\section{Novel laparoscopic technique permits 'scar-free' donor nephrectomy}

Natural body orifices such as the vagina and rectum offer concealed access points for potentially scar-free laparoscopic surgery (socalled 'natural orifice translumenal endoscopic surgery'). Gill and colleagues from the Cleveland Clinic, Cleveland, $\mathrm{OH}$, have developed a related technique, whereby access is obtained via the umbilicus. They recently presented the first four cases of donor nephrectomy performed by use of this approach.

The first procedure was carried out on 28 November 2007. A single-access R-port was inserted into the abdomen via a $2 \mathrm{~cm}$ vertical intraumbilical incision and a $2-3 \mathrm{~cm}$ rectus fasciotomy, to enable entry of the laparoscope and instruments. After being freed, the kidney was enclosed in a polyurethane pouch and removed transumbilically, with extension of the rectus fascia incision and the skin incision as necessary.

The technique was successful in all cases; no extraumbilical incisions were required (median umbilical incision length was $4 \mathrm{~cm}$ ) and no intraoperative complications occurred. Surgery lasted a median of $3.3 \mathrm{~h}$ and kidneys were exposed to a median warm ischemia time of $6.2 \mathrm{~min}$. All kidneys functioned immediately upon implantation. Each donor remained in hospital for 3 days.

Scar-free surgery is likely to appeal to living kidney donors. The authors contend that their technique might be preferable to the original translumenal approach because the transumbilical technique is less technically challenging and does not require opening a healthy organ to access a diseased organ. A prospective study comparing single-port transumbilical donor nephrectomy with the conventional laparoscopic procedure is currently underway.

Original article Gill IS et al. (2008) Single port transumbilical (E-NOTES) donor nephrectomy. J Urol 180: 637-641

\section{PKA overexpression predicts treatment outcome in men with prostate cancer}

Protein kinase A type I (PKA) mediates cell processes such as DNA replication and cell proliferation in mammalian cells. Overexpression of PKA is associated with poor prognosis in studies of patients with breast, colon and lung cancers. Khor and colleagues have now investigated the predictive value of PKA overexpression in men with prostate cancer.

Both manual and image-analysis methods were used to score the immunohistochemical staining intensity of PKA expression in pretreatment biopsies obtained from 80 men who participated in the phase III, randomized, Radiation Therapy Oncology Group (RTOG) trial 86-10. This trial compared the effects of radiotherapy alone with radiotherapy plus short-term androgen deprivation therapy in men with prostate cancer.

On univariate analysis, significant associations were identified between PKA overexpression and cause-specific mortality ( $P=0.037$ scored manually and $P=0.014$ scored by image analysis), distant metastasis $(P=0.029)$, local failure $(P=0.011)$ and biochemical failure $(P=0.022)$. On multivariate analysis, the relationships between PKA overexpression and biochemical failure $(P=0.03)$, local failure $(P=0.002)$ and distant metastasis $(P=0.018)$ remained significant.

In this small group of men with prostate cancer, PKA overexpression seemed to be a stronger determinant of outcome after radiotherapy than the addition of short-term androgen deprivation therapy was. The authors suggest that PKA overexpression could be a potentially useful biomarker for patients with high-risk prostate cancer, but further studies are needed to confirm these results.

Original article Khor L-Y et al. (2008) Protein kinase A RI- $\alpha$ predicts for prostate cancer outcome: analysis of radiation therapy oncology group trial 86-10. Int J Radiat Oncol Biol Phys 71: 1309-1315 